\title{
Improving Glycemic Control in Children with Diabetes through Implementation of Multidisciplinary Team Approach
}

\author{
Deeb Asma*, Attia Salima, Yousef Hana, Abdelrahman Layla, Suliman Shaker and Tomy Mary \\ Department of Paediatric Endocrinology, Mafraq Hospital, P O Box 2951, Abu Dhabi, United Arab Emirates
}

Received: April 23, 2016; Accepted: May 24, 2016; Published: May 27, 2016

*Corresponding author: Deeb Asma, Department of Paediatric Endocrinology, Mafraq Hospital, P O Box 2951, Abu Dhabi, United Arab Emirates, Tel: +971-50-8350568; Fax: +971-2- 5012199; E-mail: adeeb@mafraqhospital.ae

\begin{abstract}
Diabetes Mellitus is one of the most common Paediatric metabolic disorders with an incidence that is rapidly rising worldwide. Its management requires a multidisciplinary approach with involvement of highly specialized team members. Lack of proper diabetes management in children and adolescents results in poor glycemic control and predisposes to acute and long term complications. We aim to study the impact of implementing a multidisciplinary team approach on the glycemic control in children and adolescents with diabetes.
\end{abstract}

We have built a multidisciplinary team over a period of 4 years and examined the impact of provision of diabetes management on the diabetes control of the children and adolescents attending our diabetes department. Data of HbA1c of patients followed in the department and rate of admission for DKA was collected over 4 years' period. Average $\mathrm{HbA1c}$ and rate of admission for DKA were drawn up annually over the study period. First year of the study was considered the baseline point with which mean annual HbA1c was compared.

A multidisciplinary team of 8 members was built over the study period. Total number of patients in clinic increased from 37 to 79, 190, 272 and 331 in years 2010 to 2014 respectively. Average (mean) age for patients in clinic was 1.2-18.7 years (9.4) with a 1:1 gender ratio.

Percentage of patients with $\mathrm{HbA} 1 \mathrm{c}$ less than $7.5 \%$ rose from 0 to 6,13 and $21 \%$ from clinic population on yearly basis from 2011 to 2014. For the category of A1c between 7.5-9.5\%, percentage of patients increased from 40 to 45,59 and $61 \%$ over years between 2011 to 2014 ( $P=0.002$ ). Number of patients with HbA1c above $9.5 \%$ dropped from 60 to 49,28 and $18 \%$ annually by $2014(\mathrm{P}=0.003)$. Rate of DKA admission decreased significantly over the period of study ( $\mathrm{P}$ $=0.001$ ).

Implementing a multidisciplinary approach in treating children and adolescents with diabetes can result in improving glycemic control. The approach is proven to be effective and sustainable.

\section{Introduction}

Diabetes Mellitus (DM) is one of the most common Paediatric metabolic disorders affecting almost 1.7/1000 children below 18 years of age [1]. Its incidence is rapidly increasing worldwide by a rate of $3 \%$ to $5 \%[2]$.
The management of type $1 \mathrm{DM}$ requires high adherence to complex tasks such as insulin injection, blood glucose monitoring, nutrition plans and physical activity [3]. Guidelines have recently changed to encourage an HbA1c in children of less than 7.5\% [4]. To fulfill the requirements for proper management and control, a well-structured plan at the levels of hospital and community is required. Due to the fact that DM is a chronic complex disease, creation of multidisciplinary facilities offering specialized care service for its management is essential, as it is known to improve glycemic control and quality of life in patients with diabetes [5]. The subspecialty care offered by the multidisciplinary team is proven to be directly related to better diabetes outcomes and reduction of complications [6]. Amongst those complication is Diabetes Ketoacidosis (DKA) which is estimated to occur in $10 \%$ of the type 1 established population [7].

Specialized care is essential for management of chronic diseases like diabetes. This is particularly the case in children as their physical, developmental and emotional needs are complex. Caring for such needs through multidisciplinary teams in specialized centers ensures the best long-term outcomes [8]. It is proven that ensuring high family education and competence in the disease management is associated with better emotional well-being. Intensive education and treatment may also prevent or delay the onset and progression of complication [9].

Prior to the project, there was a lack of multidisciplinary team to provide care for children and adolescents with diabetes. Patients were managed by general physicians and nonspecialized nurses. There was no diabetes dietetic or psychology service. Use of mixed insulin's was common and insulin pumps were not available.

The project was a quality improvement initiative to improve care for children and adolescents with diabetes and expand diabetes service provided by the team.

\section{Aim}

The project aims to study if implementation of a multidisciplinary team approach results in improving glycemic control in children and adolescents with type 1 diabetes. 


\section{Patients and Method}

At the start of the project, comprehensive gap analysis was done to identify gaps in the care provided to children with diabetes in the current system. The analysis identified lack of a multidisciplinary team to manage children with type 1 diabetes in compliance with the international guidelines for diabetes management. The planned healthcare model was to build a multidisciplinary team, which was commenced in 2011 and completed in 2014.

Improvement of glycemic control in the patients was chosen to be the key performance indicator for the newly developed team and the primary end-point for the project. Reduction of diabetes ketoacidosis was a secondary end-point. The HbA1c monitoring is part of diabetes standard of care and is done in a central hospital lab at 8-12 weekly interval for all patients diagnosed with diabetes. Database of patients with diabetes under 18 years of ages who are in regular follow up at the diabetes unit was constructed. Lists of patients were drawn on yearly basis. Health Information department in the hospital was approached to pull out figures of HbA1c for each patient for each year's list. Yearly average of HbA1c for all clinic patients is calculated. The baseline measurement was taken as the average HbA1c for the year between 2010-2011 prior to establishment of the diabetes multidisciplinary team. Onwards, annual average HbA1c of the clinic was obtained. Comparison of the HbA1c average during 2010-2011 (baseline) with the following 4 years' annual average was done.

Number of patients admitted to hospital for DKA per 100 patients followed up in clinic was calculated on yearly basis. Comparison was performed between the baseline figure and the consequent years' figures.

\section{Data stratification}

HbA1c for all patients under 18 years was obtained. Annual average $\mathrm{HbA1}$ c for all clinic population was claculated for 4 years period. HbA1c was subdivided into 3 categories:

1. HbA1c less than $7.5 \%$ (well-controlled)

2. HbA1c 7.5-9.5 (suboptimal control)

3. HbA1c above 9.5 (poor control)

\section{Intervention}

A multidisciplinary team for management of children with diabetes was built. Specialist diabetes nurses and dieticians received intensive training in Paediatric diabetes management. Educators received special training on the use of technology in treatment of diabetes. The Paediatric diabetes caseload was divided between the 4 diabetes educators. One diabetes educator was promoted as the Paediatric diabetes service coordinator and was in charge of maintaining and updating the clinic database. She also has the task of allocating patients to educators based on number of patients per educator, preferred gender of educator by family, language spoken and special interest of the educator in relation to treatment technology used per each patient.
Each patient was allocated a named diabetes educator to be the first line to access of service and was provided with open access to diabetes clinic. Patients were provided with direct contact number of the named-educator after hours. Each educator offered 24/7 telephone access to his/her group of patients. The role of the educators included introduction of initial and ongoing patient education, setting up group education meetings, training patients at a high level on devices and equipment used to manage diabetes, conducting awareness campaigns for diabetes. Patients and families were educated on the importance of patient-team collaboration to prevent and treat diabetes complication. They were, also, engaged in various special education programs and involved in departmental surveys and feedback. Patients were encouraged to, closely, monitor their glucose profile and educated on the importance of keeping a good glycemic control. Education on DKA recognition, prevention and management received a high priority in patients and family education. Educators used various audiovisual methods to explain the condition to patients who were given written and digital material for consolidating the knowledge they gain from education sessions. Patients are also trained on using blood and urine ketone strips to detect early signs of DKA. Patients were instructed on the level at which management of ketosis should be started. Detailed instruction on how to avoid and treat DKA was given special attention to patients on insulin pump therapy.

\section{Statistical analysis}

Chi Square test was performed to test the difference in average HBA1c and admission rate through the study period. $\mathrm{P}$ value was considered significant if it was found to be less than 0.05 .

\section{Results}

A multidisciplinary team built consisted of 3 physicians (one consultant and 2 specialists), 2 diabetes educators, 2 dieticians and one psychologist. Total number of patients in clinic increased from 37 to 79, 190, 272 and 331 in years 2010 to 2014 respectively. Average (mean) age for patients in clinic was 1.2-18.7 (9.4) years with a gender ratio of approximately 1:1 male to female. $50 \%$ of patients were Arab Emirati with the rest consisting of other Arab (non-Emirati), Asian (Indian, Pakistani) and Europeans.

Percentage of patients with $\mathrm{HbA} 1 \mathrm{c}$ less than $7.5 \%$ rose from 0 to 6,13 and $21 \%$ for clinic population on yearly basis from 2011 to 2014. This observation was statistically significant with a $\mathrm{P}$ value of 0.002 . For the category of A1c between $7.5-9.5 \%$,

Table 1: Percentage of HbA1c in different categories during the study years.

\begin{tabular}{|c|c|c|c|c|}
\hline $\begin{array}{l}\text { HbA1c } \\
\text { Category }\end{array}$ & 2011 & 2012 & 2013 & 2014 \\
\hline $\begin{array}{l}\text { Less than } \\
7.5 \%\end{array}$ & 0 & 6 & 13 & 21 \\
\hline $7.5-9.5 \%$ & 40 & 45 & 59 & 61 \\
\hline $\begin{array}{l}\text { Above } \\
9.5 \%\end{array}$ & 60 & 49 & 28 & 18 \\
\hline
\end{tabular}




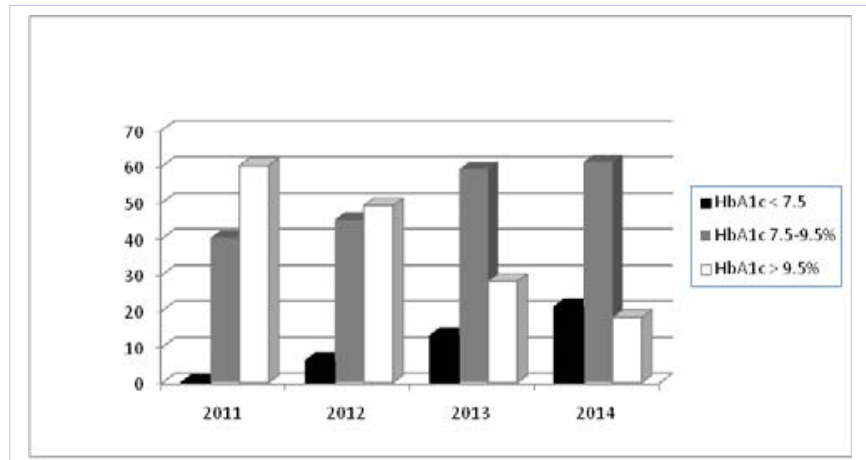

Figure 1: Percentage of patients' HbA1c in each category for years between 2011-2014.

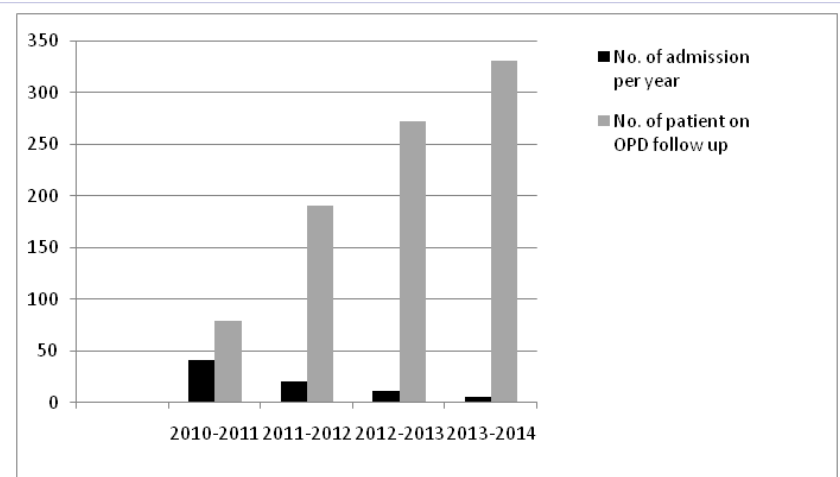

Figure 2: Number of patients admitted for DKA with total patient number followed up in the outpatient department.

percentage of patients increased from 40 to 45,59 and $61 \%$ over years between 2011 to 2014, this resulted in a decrease of the percentage of patients with HbA1c above 9.5 from $60 \%$ in year 2011 to 49, 28 and $18 \%$ for 2012, 2013 and 2014 respectively ( $P$ $=0.003$ ) (table \& figure 1 ).

Acute admission for DKA dropped from 78 episodes for the year 2010 to 41, 21, 12 and 6 episodes for years 2011-2014 respectively $(P=0.001)$ (figure 2$)$.

Over the study period, insulin pump use has increased to $50 \%$ in 2014 when half of the patients used insulin pumps and the other half was managed on Multiple Daily Injections (MDI) of insulin. $60 \%$ of pump users used sensor-augmented pump version and a third utilized the hypoglycemia automatic off switch pumps.

\section{Discussion}

A multidisciplinary Paediatric diabetes team can provide cost effective care to children with diabetes. The effective care results from improving metabolic outcomes, delaying and preventing complication. Lack of proper patient and family education and management of a multidisciplinary team leads to increase risk of complications [6]. In our hospital, diabetes care for children and adolescents with diabetes was originally delivered by general Paediatric team of physicians and nurses with no specialized expertise in Paediatric diabetes management. A strategic plan was put from the outset of the project to ensure provision of all requirements for service. Special emphasis was put to offer comprehensive education for patients provided by highly qualified multidisciplinary team.

A direct impact of improved metabolic control will be fewer hospitalizations for acute complications [10]. In particular, DKA is an expensive complication of type 1 diabetes with a mean cost per hospitalization of $\$ 10,876 \pm 11,024$ [11]. We have shown a significant reduction of acute admission due to DKA following implementing the model of multidisciplinary team approach [12].

The Diabetes Control and Complication Trial (DCCT) provided clear evidence in adults and adolescents that improved metabolic control, is associated with fewer and delayed microvascular complications [13]. Follow-up data from the DCCT through the Epidemiology of Diabetes Interventions and Complications (EDIC) trial indicates those 5-7 years of poor glycaemic control during adolescence and young adulthood, results in an increased risk for microvascular and macrovascular complications in the subsequent 6-10 years [14].

HbA1c monitoring has been shown to be the most useful measure in evaluating metabolic control and is the only measure for which good data are available in terms of its relationship with later microvascular and macrovascular complications [13]. Our study showed a significant increase in the number of patients who achieved the international target of diabetes control [4].

Dieticians, diabetes nurse educators and clinical psychologist are valuable members of the diabetes multidisciplinary team. Dietician offer medical nutrition therapy, which is necessary for a successful management of type 1 diabetes [15]. Accordingly, dietetic support constitutes a major part of diabetes service. Creating positions for dieticians and their regular development is essential. All children with type 1 diabetes should receive counselling from a registered dietitian experienced in Paediatric diabetes. Equally important is provision of diabetes education by competent diabetes nurses. Patient and family education is a major step in coping with diabetes management. Our team consisted of experienced dieticians and diabetes educators who were all certified pump trainers. Continued education of staff employed was maintained as a priority. Provision of advanced management resources for treating children under the care team was ensured. Required devices utilizing advanced technology were provided and education on their use was made available by a team of expert educators.

This development enabled us to utilize advanced forms of insulin pump to improve diabetes control in our patients.

Diagnosis of type 1 diabetes in a child imposes a big burden on families and the initial phase following diagnosis is linked with various emotional and psychological reactions. It is shown in many studies that encouraging caregivers to discuss emotional and physical concerns by competent psychologists is reflected positively on the initial reaction to disease diagnosis and longterm control [16]. Our psychologist was a trained psychotherapist and offered counseling for patients and families at the initial 
diagnosis and onwards.

There aremanylimitations of implementing a multidisciplinary approach for treatment of diabetes in children and adolescents. This highly specialized area requires subspecialty training of various health care providers. Lack of dieticians highly trained in diabetes management is a big obstacle in establishing a high quality diabetes center. Specialist nurses are hard to recruit and need continued training to keep up to the moving field of technology. Psychology service is not widely available and experienced psychologists in management of chronic disease need special qualification and training. Shortage of staff is a main issue amongst facilities, which run diabetes service for children and adolescents. Lack of budget to fund various position is a major obstacle.

It is known that health care costs for type 1 diabetes patients are higher than those for general population [17]. Insurance can be a challenge, too. In the SEARH of diabetes youth study, lack of private health insurance was found to be a risk factor in development of DKA [18]. Implementing and sustaining the diabetes care model can be hard to achieve in institution with limited resources.

Majority of insurer in our region do not cover glucose, ketone strips, insulin pump and continuous glucose monitoring devices and their accessories. Lack of insurance cover results in limited access to care and inability to use advanced technology in our region (personal experience).

\section{Conclusion}

As glycemic control is a major contributor to long-term, diabetes complications, investing in building a multidisciplinary team improve not only short term control but also prevent chronic complications. Implementing a multidisciplinary approach in treating young people results in improving care and improving glycemic control. However, its implementation imposes various challenges.

\section{Acknowledgement}

We acknowledge Miss Helga Springhorn, Miss Hala Disi from Mafraq hospital Quality team department for their support.

\section{Ethical Approval}

This project is deemed as an improvement project. Ethical approval was not required.

\section{References}

1. Center of disease control (CDC): statement on type diabetes prevalence. 2005.

2. Wodrich DL, Hasan K, Parent K B. Type 1 diabetes mellitus and school: a review. Pediatr Diabetes. 2011;12(1):63-70. doi: 10.1111/j.13995448.2010.00654.x.

3. Hains AA, Berlin KS, Davies WH, Sato AF, Smothers MK, Clifford LC, et al. Attribution of teacher reactions to diabetes self-care behaviors. J Pediatr Psychol. 2009;34(1):97-107. doi: 10.1093/jpepsy/jsn041.

4. Acerini C, Craig ME, de Beaufort C, Maahs DM, Hanas R. International
Society for Paediatric and Adolescent Diabetes (ISPAD) Clinical Practice Consensus Guidelines. Paediatric Diabetes. 2014;15:S20.

5. Codispoti C, Douglas MR, McCallister T, Zuniga A. The use of a multidisciplinary team care approach to improve glycemic control and quality of life by the prevention of complications among diabetic patients. J Okla State Med Assoc. 2004;97(5):201-4.

6. Coblin RH. Subspecialist care improves diabetes outcomes. Diabetes Care. 2002;25(9):1654-6.

7. Rewers A, Chase HP, Mackenzie T, Walravens P, Roback M, Rewers $\mathrm{M}$, et al. Predictors of acute complications in children with type 1 diabetes. JAMA. 2002;287(19):2511-2518.

8. von Sengbusch S, Müller-Godeffroy E, Häger S, Reintjes R, Hiort O, Wagner V. Mobile diabetes education and care: intervention for children and young people with Type 1 diabetes in rural areas of northern Germany. Diabet Med. 2006;23(2):122-127.

9. Graue M, Wentzel-Larsen T, Bru E, Hanestad BR, Sovik O. The coping styles of adolescents with type 1 diabetes are associated with degree of metabolic control. Diabetes Care. 2004;27:1313-1317.

10. Paris CA, Imperatore G, Klingensmith GJ. Predictors of insulin regimens and impact on outcomes in youth with type 1 diabetes: The SEARCH for diabetes in youth study. J Pediatr. 2009;155:183-189.

11. Maldonado MR, Chong ER, Oehli MA, Balasubramanyam A. Economic Impact of Diabetic Ketoacidosis in a Multiethnic Indigent Population Analysis of costs based on the precipitating cause. Diabetes Care 2003;26(4):1265-1269.

12. Deeb A, Yousef H, Abdelrahman L, Tomy M, Suliman S, et al. Implementation of a diabetes educator-care model to reduce paediatric admission for diabetic ketoacidosis. J Diabetes Res. 2016. 2016.

13. The effect of intensive treatment of diabetes on the development and progression of long term complications in insulin-dependent diabetes mellitus. The Diabetes Control and Complications Trial Research Group. The Diabetes Control and Complications Trial Research Group. N Engl J Med. 1993;329:977-986.

14. Nathan DM, Lachin J, Cleary P, Orchard T, Brillon DJ, Backlund JY, O'Leary DH, et al. Intensive diabetes therapy and carotid intima-media thickness in type 1 diabetes mellitus. N Engl J Med 2003;348:22942303.

15. Bantle JP, Wylie-Rosette J, Albright AL, American Diabetes Association. Nutrition recommendations and interventions for diabetes: A position statement of ADA. Diabetes Care. 2008;31 Suppl 1: S61-78. doi: $10.2337 /$ dc08-S061.

16. Olsen Roper S, Call A, Leishman J, Ratcliffe GC, Mandleco BL, Dyches TT, et al. Type 1 diabetes: Children and adolescents' knowledge and questions. J Adv Nurs. 2009;65(8):1705-14. doi: 10.1111/j.13652648.2009.05033.x.

17. Angus VC, Waugh N. Hospital admission patterns subsequent to diagnosis of type 1 diabetes in children: a systematic review. BMC Health Serv Res. 2007;5(7):199.

18. Dabelea D, Rewers A, Stafford JM, Standiford DA, Lawrence JM, Saydah $S$, et al. Trends in the prevalence of ketoacidosis at diabetes diagnosis: the SEARCH for diabetes in youth study. Paediatrics 2014;133(4):938945. 